\title{
Impact of an IR-UWB Reading Approach on Chipless RFID Tag
}

\author{
Marco Garbati, Student Member, IEEE, Romain Siragusa, Etienne Perret, Senior Member, IEEE, \\ and Christophe Halopé
}

\begin{abstract}
An unusual configuration for chipless radio frequency identification (RFID) reader based on impulse radio ultrawideband (IR-UWB) is proposed. The impact of this approach on chipless tag reading performance, compared to frequency modulated continuous-wave (FMCW) techniques is studied. By reducing the inner sampling clock jitter of the proposed reader, it is possible to have resolution comparable to the one obtained with laboratory equipment.
\end{abstract}

Index Terms-Chipless RFID reader, IR-UWB radar, jitter, radiofrequency identification, sampling methods.

\section{INTRODUCTION}

$\mathbf{O}$ NLY few articles focusing on chipless radio frequency identification (RFID) readers can be found in literature, while the reader plays a significant role in application based on chipless RFID technology. In [1] the authors present a reader based on ultra-wideband (UWB) chirp signal interrogation. The hardware architecture is close to a frequency modulated continuous-wave (FMCW) radar. It has a UWB voltage controlled oscillator (VCO) in transmission, and a down conversion process in reception. In [2] a customized reader for dual polarized tags is presented, its architecture is close to [1] with the duplication of some hardware parts for cross polarization reading capability. In [3] a compact and low cost reader based on FMCW is also presented. All the proposed reader solutions present strong limitations to be used in practical applications. First, they are based on FMCW approach, and due to the Federal Communications Commission (FCC) or even the European Telecommunications Standards Institute (ETSI) UWB mean power spectral density (PSD) over $\mathrm{MHz}$ transmitting limitations for indoor applications, it would be extremely difficult to read chipless tags in practical environment [4]. Second, the reading time could easily exceed $1 \mathrm{~s}$ due to the reading sweep time which is directly proportional to the reader bandwidth. In [1] a reading time estimation of $500 \mathrm{~ms}$ is given for one row acquisition (no averaging) with a bandwidth of $2.2 \mathrm{GHz}$. But the post processing treatment time, and an eventual data transmission to a central unit needs to be added. In case of sweep averaging process, the reading time could reach several tens of seconds. In [3] the sweep time is only $10 \mathrm{~ms}$, but with a bandwidth limited to $1.25 \mathrm{GHz}$.

Marco Garbati, Romain Siragusa and Etienne Perret are with Laboratoire de Conception et dIntgration des Systmes (LCIS), Grenoble Institute of Technology (Grenoble INP), Valence 26902, France. Etienne Perret is also with Institut Universitaire de France, Paris, France. Christophe Halopé is with Arjowiggins Security, Apprieu, France. (marco.garbati@lcis.grenoble-inp.fr, romain.siragusa@1cis.grenoble-inp.fr, etienne.perret@lcis.grenoble-inp.fr, Christophe.Halope@arjowiggins.com).
To overcome these problems, readers based on IR-UWB and equivalent time, where the tags are interrogated with subnanosecond impulses, have been proposed by the authors [5], [6]. They have an input bandwidth respectively of $3.1-5.5 \mathrm{GHz}$ and 3.1-8 GHz and they operate in the UWB regulation and standard where all the energy transmitted towards the tag is concentrated in a sub-nanosecond pulse. This allow a higher instantaneous trasmitting power compared to UWB FMCW. Moreover, the absence of frequency sweep in transmission allows a smaller reading time, and a real-time acquisition can even be obtained. The reader proposed in this article has a reading time ranging from $6 \mathrm{~ms}$ to $50 \mathrm{~ms}$ depending on the averaging, respectively for 1 and 64, and has a minimum equivalent sampling period of $10 \mathrm{ps}$. Note that a shorter reading time is possible using expensive real time laboratory equipment. The aim of this article is to compare IR-UWB and FMCW approach in term of reading time, chipless tag reading accuracy and robustness of phase noise (i.e. Jitter). The second section presents the improved IR-UWB reader prototype. The third section deals with the comparison of both approaches. Finally some conclusions are drawn.

\section{UWB CHIPLESS RFID READER PROTOTYPE}

The chipless RFID reading system based on IR-UWB approach, taken as reference for the study, employs laboratory equipment. It is composed of: 1) an impulse generator which transmits a sub-nanosecond UWB signal toward the tag, and 2) a real time digital signal oscilloscope (DSO) in reception. As the objective of this article is to study the effect of such reading approach compared to $\mathrm{FMCW}$, a reader prototype improved from [5], [6] has been conceived. The same hardware architecture as [6] has been used but substantial improvements, especially in terms of VHDL architecture and post-processing have been realized. These variations have allowed to reduce radically the reading time, to improve the sequential equivalent time algorithm application, and thus the robustness of tag's ID detection. The Fig. 1 shows the block schematic diagram of the proposed reader. It is composed by: 1) the triggerable HL9200 impulse generator from Hyperlabs, 2) the Spartan 6 FPGA SP601 evaluation board from XilinX, which represents the reader control unit (CU), 3) a 7x7 $\mathrm{cm}^{2}$ designed mixed-signal acquisition board. The latter presents two SMA connectors, one for the receiving antenna, i.e. the response of the tag, and the other one to trigger the impulse generator. The reader communicates with a computer through universal serial bus (USB) connection with the RS232 universal asynchronous 


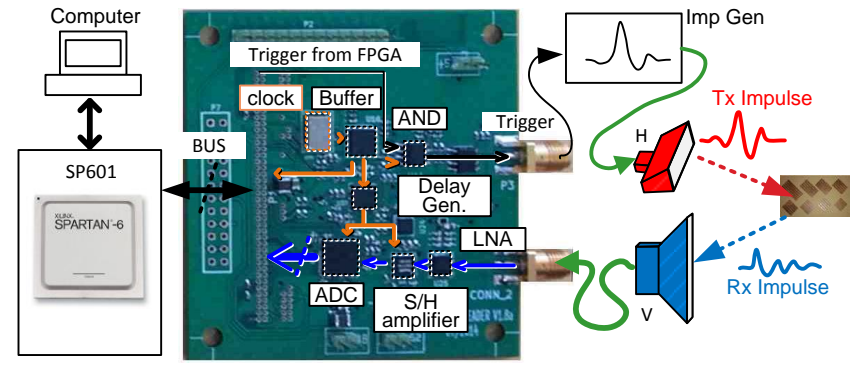

Fig. 1. IR-UWB chipless RFID reader proposed block schematic diagram.
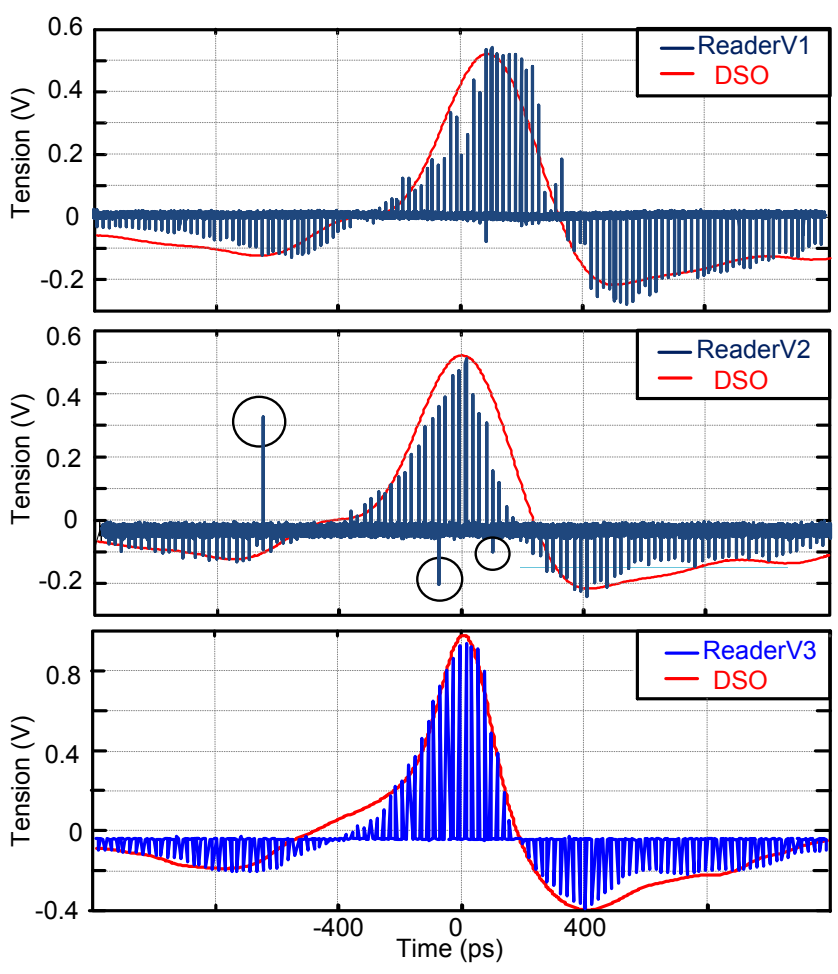

Fig. 2. Time acquisition of the impulse without any averaging. The acquisitions have been performed with different reader versions and with the DSO.

receiver-transmitter (UART) $921.16 \mathrm{~kb} / \mathrm{s}$ protocol. The mixed board has in reception a LNA Hittite HMC753LP4E, followed by the Inphi $1321 \mathrm{TH}$ sampling and holding amplifier, hereafter called the sampler, and the low-cost AD9115 ADC from Analog Devices. The sampler is Commercial Off-the-Shelf (COTS) and, for UWB signals, its cost is lower than $1 \mathrm{k} €$. Such component is able to sample an UWB (3.1-10.6 GHz) signal, with a low sampling clock, giving the time to the ADC, which is low-speed, to convert numerically the sample.

By connecting the impulse generator output to the reader input, acquisitions with the different versions of our reader have been performed. Te results are shown in Fig. 2. To underline the reading performance, the measures were performed without averaging with the three versions. ReaderV1 and ReaderV2 have been introduced respectively in [5] and [6]; ReaderV3 is the currently presented version. The ReaderV2 shows a smaller sampling noise compared to ReaderV1. This was achieved with specific improvements based on the hardware architecture to reduce significantly the overall system jitter. Anyway this version V2 presents some problems of synchronization. Indeed as underlined with circles in the Fig. 2, some acquired points are referred to a different timing position. This problem has been corrected in the last version thanks to the implementation of customized synchronization blocks at VHDL level. Contrarily to the earlier versions, ReaderV3 presents an equivalent time sampling period which can be a multiple of $10 \mathrm{ps}$. Moreover the reading frame has a variable duration from 8 ns to $64 \mathrm{~ns}$. The pulse repetition frequency (PRF) is about $1 \mathrm{MHz}(1 \mu \mathrm{s}$ period). The reading time depends from the chosen parameters, for example, it is $6 \mathrm{~ms}$ without averaging. It represents a huge improvement comparing to the architectures presented in [1]-[3], but also with the latest versions, which was in order of tens of seconds depending on the chosen averaging factor. This remarkable amelioration is due to the realization of a customized VHDL block which was able to perform a hardware averaging during the acquisition. Note that the acquisition would take hundreds of milliseconds with an easier software solution.

\section{IR-UWB VS FMCW AND JITTER INFLUENCE}

A FMCW approach gives the possibility to perform more accurate measurement compared to an IR-UWB solution. This is because of the higher dynamic that can be obtained with such solution. The interrogating signal is sweeping around the frequency band of the tag, where the sweep time is defined at the reader level. Conversely in IR-UWB systems, the interrogating signal is a short pulse in the UWB band, and the tag response lasts notably for only few nanoseconds (around $12 \mathrm{~ns}$ ) [7]. As we are going to see, because of the short duration of the tag response and its amplitude envelope, an IR-UWB can perform tag reading with a limited frequency resolution compared to a FMCW radar. Also, an IR-UWB systems is affected by sampling clock jitter. Its impact is more significant at higher frequency, where a small unwanted timing translation of the sampling clock event corresponds to a higher phase variation of the acquiring signal. To quantify this impact, an equivalent time reading system with different parameters has been emulated in Matlab, and the results are shown in Fig. 3. An ideal tag mode response, where the tag is supposed to have eight resonances between 3.2 and $6.7 \mathrm{GHz}$ like in [4], with a infinitive quality factor, has been emulated. Two different tag's responses duration (100 ns and 12 ns) have been considered, and a Hamming window has been applied. Moreover in case of $12 \mathrm{~ns}$, the reading system was supposed to be affected by jitter of $0,7.34$ and $40 \mathrm{ps}$ respectively. Analyzing the effect of the two different signal durations without jitter, the larger one allows a much better reading frequency resolution, which can be translated in the possibility of developing tags with smaller inter-distance between resonances and therefore higher bit capacity. The larger duration corresponds to the case of a FMCW interrogation, where the IF bandwidth can be in orders of $\mathrm{kHz}$. We also have studied the effect of the jitter variation for the smaller response duration, which is representative to a real tag signal in case of IR-UWB based interrogation system. The jitter variation affects the reading capability because the distance between the 

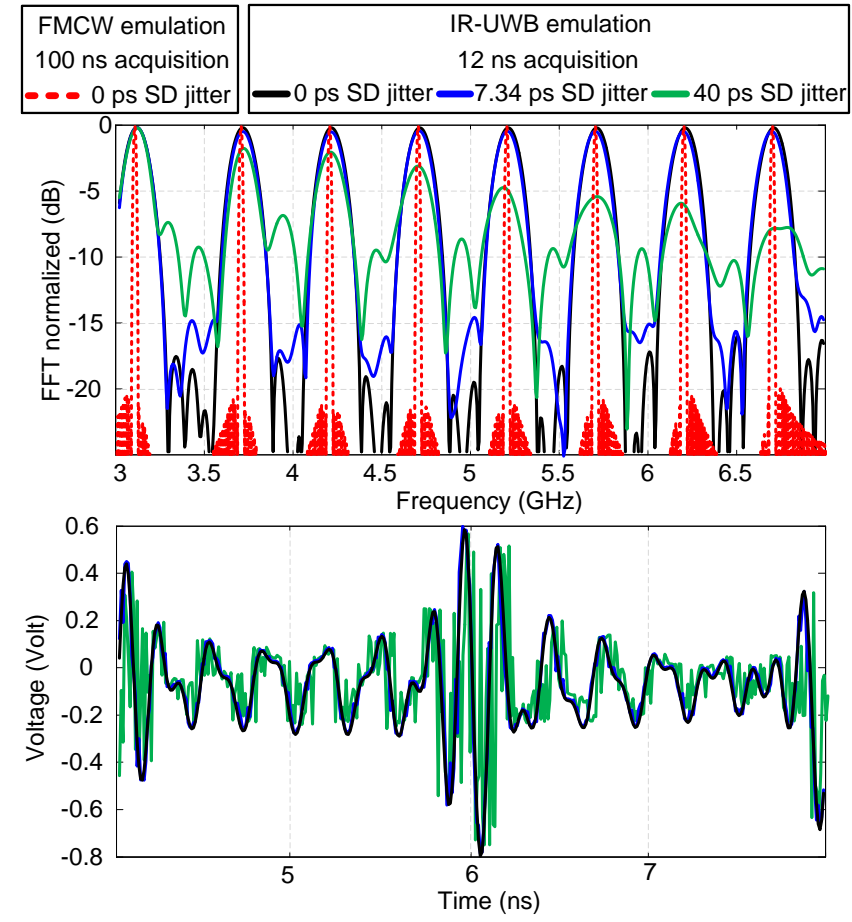

Fig. 3. Emulation of readers based on FMCW and IR-UWB with variable jitter.

main lobs of the tag's response (which are located around the tag's frequency resonants), and the secondary lobs is getting smaller when the jitter increases. Moreover the peak apexes corresponding to these main lobs decrease with frequency. We note that the frequency of these peak apexes and the bandwidth of these lobs also vary especially at higher frequency.

Fig. 4 shows a chipless tag acquisition with different reading systems which are based on FMCW and IR-UWB with different performance in terms of jitter. The frequency domain chipless tag employed presents eight resonances between 3 and $7 \mathrm{GHz}$ [5]. All the measurement were performed in anechoic chamber to reduce as much as possible the noise distinct from the one caused by the jitter system. The measurement with the VNA N5222A emulates a chipless reader based on FMCW. The DSO 91204A measurement was performed with the Hyperlabs HL9200 impulse generator in a IR-UWB configuration. As expected the measurement with the VNA presents the best performance and with the bigger frequency resolution. In addition compared to the others measurement (IR-UWB configurations), all the peaks present a comparable power response. This is due to the approach, in case of IR-UWB, the impulse generator frequency response is not flat for all the UWB band. Nevertheless the measurement performed with the readerV3 and the DSO are similar and fully successful. Finally the readerV1, which has a larger jitter of $7.34 \mathrm{ps}$, presents the worst reading performance. It is not possible to read correctly the resonance positions at higher frequency and the others presents shift in frequency of few MHz. This drop of performances is mostly due to the effect of system jitter, also play an important rule the noise generated by the inefficient application of the equivalent time

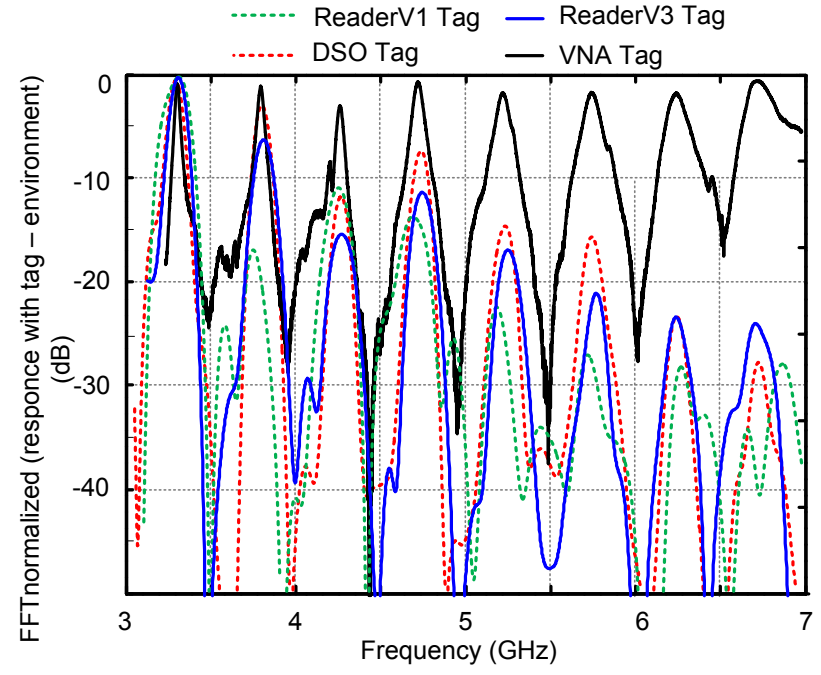

Fig. 4. Measurements in protected environment different reading systems.

algorithm due to synchronization issue. It can add noise in the entire tag's bandwidth, and its negative effects are more important at higher frequency where the tag's response has lower amplitude.

\section{CONCLUSION}

The IR-UWB approach is the preferable reading architecture, according to the rules in terms of UWB emissions, and in terms of reading time. Anyway inner performance obtained from such solutions is lower than with a FMCW reading, and an internal jitter of few ps can reduce significantly the chipless tag reading performance. A reader based on IRUWB optimized for reducing jitter and reading time has been presented. Currently, it is the only solution that can be compatible with chipless RFID applications according to UWB emission regulation and reading time.

\section{REFERENCES}

[1] R.V. Koswatta and N.C. Karmakar, "A Novel Reader Architecture Based on UWB Chirp Signal Interrogation for Multiresonator-Based Chipless RFID Tag Reading," in IEEE Trans. Microw. Theory Techn., vol. 60, no. 9, pp. 2925-2933, Sept. 2012.

[2] M.A. Islam, A. Azad and N. Karmakar, "A novel reader architecture for chipless RFID tags," Asia-Pacific Symposium on Electromagnetic Compatibility (APEMC), pp. 1-4, 2013.

[3] M. Ppperl, C. Carlowitz, M. Vossiek, C. Mandel and R. Jakoby, "An ultra-wideband time domain reflectometry chipless RFID system with higher order modulation schemes," in German Microwave Conf (GeMiC), pp. 401-404, 2016.

[4] A. Vena, E. Perret and S. Tedjni, A depolarizing chipless RFID tag for robust detection and its FCC compliant UWB reading system, in IEEE Trans. Microw. Theory Techn., vol. T61, no. T8, pp. T2982-2994, August T2013.

[5] M. Garbati, R. Siragusa, E. Perret, A. Vena and C. Halop, "High performance chipless RFID reader based on IR-UWB technology," in 2015 9th European Conference on Antennas and Propagation (EuCAP), pp. $1-5,2015$

[6] M. Garbati, R. Siragusa, E. Perret and C. Halop, ”Low cost low sampling noise UWB Chipless RFID reader," in 2015 IEEE MTT-S International Microwave Symposium, pp. 1-4, 2015.

[7] A. Ramos, E. Perret, O. Rance, S. Tedjini, A. Lzaro and D Girbau, "Temporal Separation Detection for Chipless Depolarizing FrequencyCoded RFID," in IEEE Trans. Microw. Theory Techn., vol. 64, no. 7, pp. 2326-2337, July 2016. 